\title{
Abundant Extraterrestrial Amino Acids in the Primitive CM Carbonaceous Chondrite Asuka 12236
}

Daniel P. Glavin ${ }^{1}$, Hannah L. McLain ${ }^{1,2}$, Jason P. Dworkin ${ }^{1}$, Eric T. Parker ${ }^{1}$, Jamie E. Elsila ${ }^{1}$, José C. Aponte ${ }^{1,2}$, Danielle N. Simkus $^{1,3}$, Chad I. Pozarycki ${ }^{1,4}$, Heather V. Graham ${ }^{1,2}$, Larry R. Nittler ${ }^{5}$, and Conel M.O'D. Alexander ${ }^{5}$

${ }^{1}$ NASA Goddard Space Flight Center, Greenbelt, Maryland 20771, USA

${ }^{2}$ Catholic University of America, Washington, D.C. 20064, USA

${ }^{3}$ NASA Postdoctoral Program, USRA, Columbia, Maryland 20146, USA

${ }^{4}$ Southeastern Universities Research Association, Washington, D.C. 20005, USA

${ }^{5}$ Earth and Planets Laboratory, Carnegie Institution of Washington, Washington, D.C. 20015, USA

\begin{abstract}
The Asuka (A) 12236 meteorite has recently been classified as a CM carbonaceous chondrite of petrologic type 2.9 and is among the most primitive CM meteorites studied to date (Kimura et al. 2020). We report the concentrations, relative distributions, and enantiomeric ratios of amino acids in a hot water extract of the A 12236 meteorite determined by ultra-high-performance liquid chromatography time-of-flight mass spectrometry (Glavin et al. 2020). A wide diversity of twoto six-carbon aliphatic primary amino acids were identified in A 12236 , which had a total amino acid abundance of $360 \pm 18$ nmol $\mathrm{g}^{-1}$, with most amino acids present in a free form (Table 1). The amino acid concentrations of A 12236 were double those previously measured in the CM2.7 Paris meteorite (Table 1), consistent with A 12236 being a highly primitive and unheated CM chondrite. The high relative abundance of $\alpha$-amino acids in A 12236 are also consistent with formation by a Strecker-cyanohydrin dominated synthesis during a limited early aqueous alteration phase on the CM meteorite parent body. The presence of predominantly free glycine, a near racemic mixture of alanine (D/L $~ 0.93$ to 0.96 ), and elevated abundances of several terrestrially rare non-protein amino acids including $\alpha$-aminoisobutyric acid $(\alpha$-AIB) and racemic isovaline, indicate that these amino acids in A 12236 are extraterrestrial in origin. Given a lack of evidence for biological amino acid contamination in A 12236, it is possible that some of the L-enantiomeric excesses ( $\mathrm{L}_{\mathrm{ee}} \sim 34$ to $64 \%$ ) of the protein amino acids aspartic and glutamic acids and serine are indigenous to the meteorite; however, isotopic measurements are needed for confirmation. In contrast to more aqueously altered CMs of petrologic types $\leq 2.5$, no L-isovaline excesses were detected in A 12236. This observation strengthens the hypothesis that extensive parent body aqueous activity is required to produce or amplify the large L-isovaline excesses that cannot be explained solely by exposure to circularly polarized radiation or other chiral symmetry breaking mechanisms prior to incorporation into the asteroid parent body. The fact that only L-enantiomeric excesses (and no D-excesses) have been observed in amino acids with a single asymmetric carbon in carbonaceous meteorites suggests that the origin of life on Earth or elsewhere in our solar system may have been biased toward L-amino acid homochirality from the very beginning. The return of pristine materials from the surface of carbonaceous asteroids to Earth for detailed laboratory organic analyses will be essential to advance our understanding of the origin and evolution of amino acid chiral asymmetry in the early Solar System given that all meteorites on Earth have been compromised, to some degree, by terrestrial contamination. JAXA's Hayabusa2 mission and NASA's OSIRIS-REx mission will return the first pristine samples collected from the surfaces of the carbonaceous asteroids Ryugu and Bennu. These missions will provide a unique opportunity to evaluate the effects of parent body processing on the distributions and enantiomeric abundances of amino acids and other prebiotic molecules in carbon-rich asteroids.
\end{abstract}

Table 1. Summary of the average concentrations (nmol g-1) of the two- to six-carbon amino acids in the non-hydrolyzed (free) and $6 \mathrm{M} \mathrm{HCl}$ acid-hydrolyzed (total) water extracts of the CM meteorites Asuka 12236 and Paris.

\begin{tabular}{|c|c|c|c|c|}
\hline & \multicolumn{2}{|c|}{ Asuka 12236 (CM2.9) } & \multicolumn{2}{|c|}{ Paris (CM2.7) } \\
\hline & \multicolumn{2}{|c|}{ Glavin et al. (2020) } & \multicolumn{2}{|c|}{ Martins et al. (2015) } \\
\hline Amino Acids & free & total & free & total \\
\hline \multicolumn{5}{|c|}{ Dicarboxylic Amino Acids } \\
\hline D-aspartic acid & $1.7 \pm 0.2$ & $2.4 \pm 0.1$ & $0.67 \pm 0.03$ & $1.01 \pm 0.04$ \\
\hline L-aspartic acid & $1.8 \pm 0.1$ & $4.9 \pm 0.2$ & $0.90 \pm 0.05$ & $1.50 \pm 0.04$ \\
\hline D-glutamic acid & $0.63 \pm 0.07$ & $3.1 \pm 0.2$ & $4.1 \pm 0.3$ & $5.6 \pm 0.4$ \\
\hline L-glutamic acid & $0.9 \pm 0.1$ & $8.4 \pm 0.5$ & $4.9 \pm 0.3$ & $6.7 \pm 0.5$ \\
\hline \multicolumn{5}{|c|}{ Hydroxy Amino Acids } \\
\hline D-serine & $1.23 \pm 0.05$ & $1.1 \pm 0.2$ & n.r. & n.r. \\
\hline L-serine & $2.78 \pm 0.07$ & $5.0 \pm 0.2$ & n.r. & n.r. \\
\hline D-threonine & n.d. & n.d. & n.r. & n.r. \\
\hline L-threonine & n.d. & n.d. & n.r. & n.r. \\
\hline \multicolumn{5}{|c|}{ C2 Amino Acid } \\
\hline Glycine & $155 \pm 3$ & $160 \pm 5$ & $45 \pm 2$ & $110 \pm 5$ \\
\hline \multicolumn{5}{|c|}{ C3 Amino Acids } \\
\hline$\beta$-alanine & $13.1 \pm 0.6$ & $21 \pm 1$ & $7.0 \pm 0.3$ & $14 \pm 2$ \\
\hline D-alanine & $25 \pm 1$ & $28 \pm 2$ & $3.3 \pm 0.1$ & $8.0 \pm 0.4$ \\
\hline L-alanine & $26 \pm 1$ & $30 \pm 2$ & $4.9 \pm 0.5$ & $10.8 \pm 0.5$ \\
\hline
\end{tabular}




\begin{tabular}{|c|c|c|c|c|}
\hline \multicolumn{5}{|c|}{ C4 Amino Acids } \\
\hline D,L- $\alpha$-amino- $n$-butyric acid & $15 \pm 1^{\mathrm{a}}$ & $16 \pm 2^{\mathrm{a}}$ & $1.17 \pm 0.04^{\mathrm{b}}$ & $2.97 \pm 0.07^{\mathrm{b}}$ \\
\hline D- $\beta$-amino- $n$-butyric acid & $3.35 \pm 0.07$ & $4.5 \pm 0.1$ & $<0.01$ & $<0.01$ \\
\hline L- $\beta$-amino- $n$-butyric acid & $3.2 \pm 0.1$ & $4.4 \pm 0.1$ & $<0.01$ & $<0.01$ \\
\hline$\alpha$-aminoisobutyric acid & $17.0 \pm 0.2$ & $17.9 \pm 0.4$ & $0.84 \pm 0.06$ & $3.0 \pm 0.1$ \\
\hline D,L- $\beta$-aminoisobutyric acid ${ }^{\mathrm{a}}$ & n.d. & n.d. & $<0.01$ & $<0.01$ \\
\hline $\mathrm{\gamma}$-amino- $n$-butyric acid & $2.0 \pm 0.1$ & $12.1 \pm 0.6$ & $1.2 \pm 0.1$ & $1.8 \pm 0.2$ \\
\hline \multicolumn{5}{|c|}{ C5 Amino Acids } \\
\hline D-norvaline (D-2-apa) & $2.42 \pm 0.05$ & $2.7 \pm 0.2$ & $<0.3$ & $1.32 \pm 0.03$ \\
\hline L-norvaline (L-2-apa) & $2.48 \pm 0.05$ & $2.5 \pm 0.1$ & $<0.4$ & $1.52 \pm 0.05$ \\
\hline D-isovaline (D-2-a-2-mba) & $3.9 \pm 0.3$ & $4.2 \pm 0.3$ & \multirow{2}{*}{$0.22 \pm 0.02^{\mathrm{a}}$} & $1.9 \pm 0.1$ \\
\hline L-isovaline (L-2-a-2-mba) & $4.1 \pm 0.2$ & $4.0 \pm 0.2$ & & $1.9 \pm 0.1$ \\
\hline D-valine (D-2-a-3-mba) & $5.1 \pm 0.1$ & $5.6 \pm 0.9$ & $1.86 \pm 0.07$ & $4.9 \pm 0.1$ \\
\hline L-valine (L-2-a-3-mba) & $5.7 \pm 0.2$ & $6.8 \pm 0.4$ & $1.9 \pm 0.2$ & $5.2 \pm 0.2$ \\
\hline D,L-3-apab & $1.8 \pm 0.1$ & $1.8 \pm 0.2$ & n.r. & n.r. \\
\hline D,L- and allo-3-a-2-mba ${ }^{b}$ & $1.40 \pm 0.04$ & $1.4 \pm 0.2$ & n.r. & n.r. \\
\hline 3-a-3-mba & $0.92 \pm 0.04$ & $0.8 \pm 0.1$ & n.r. & n.r. \\
\hline 3-a-2,2-dmpa & $0.98 \pm 0.05$ & $2.48 \pm 0.03$ & n.r. & n.r. \\
\hline D,L-3-a-2-epa ${ }^{a}$ & $<0.3^{\mathrm{c}}$ & $<0.3^{\mathrm{c}}$ & n.r. & n.r. \\
\hline D,L-4-apa ${ }^{a}$ & $0.46 \pm 0.07$ & $1.46 \pm 0.05$ & n.r. & n.r. \\
\hline D,L-4-a-2-mba ${ }^{a}$ & n.d. & $2.6 \pm 0.2$ & n.r. & n.r. \\
\hline D,L-4-a-3-mba ${ }^{a}$ & $0.05 \pm 0.01$ & $0.28 \pm 0.02$ & n.r. & n.r. \\
\hline 5-apa & $0.64 \pm 0.04$ & $2.7 \pm 0.3$ & n.r. & n.r. \\
\hline \multicolumn{5}{|c|}{ C6 Amino Acids } \\
\hline$\varepsilon$-amino- $n$-caproic acid (EACA) & $1.16 \pm 0.05$ & $2.00 \pm 0.04$ & $<0.01$ & $<0.01$ \\
\hline Sum $\left(n m o l g^{-1}\right)$ & $300 \pm 9$ & $360 \pm 18$ & $78 \pm 4$ & $182 \pm 10$ \\
\hline
\end{tabular}

${ }^{a}$ Enantiomers could not be separated under the chromatographic conditions.

benantiomers could be separated, but not identified individually due to the lack of optically pure standards.

'Poor chromatographic resolution prevented accurate quantification, therefore only upper limits are reported.

n.d. = amino acids identified, but concentration not determined due to interfering peaks or other analytical issues.

n.r. $=$ concentration not reported.

Acknowledgments. The material discussed here was motivated and based on work supported by NASA under Contract NNM10AA11C issued through the New Frontiers Program. D.P.G, J.E.E., H.L.M., J.C.A., E.T.P., and J.P.D. also appreciate funding support from the NASA Astrobiology Institute through award 13-13NAI7-0032 to the Goddard Center for Astrobiology, NASA's Planetary Science Division Research Program, and a grant from the Simons Foundation (SCOL award 302497 to J.P.D.). We are very grateful to Dr. Akira Yamaguchi, the Antarctic meteorite curator at the National Institute of Polar Research (NIPR) for providing the Asuka 12236 meteorite sample analyzed in this study.

\section{References}

Kimura, M., Imae, N., Komatsu, M., Barrat, J. A., Greenwood, R. C., Yamaguchi, A., and T. Noguchi, The most primitive CM chondrites, Asuka 12085, 12169, and 12236, of subtypes 3.0-2.8: Their characteristic features and classification, Polar Science, in press, doi: 10.1016/j.polar.2020.100565, 2020.

Glavin, D. P., McLain, H. L., Dworkin, J. P., Parker, E. T., Elsila, J. E., Aponte, J. C., Simkus, D. N., Pozarycki, C. I., Graham, H. V., Nittler, L. R., and C. M.O’D. Alexander, Abundant extraterrestrial amino acids in the primitive CM carbonaceous chondrite Asuka 12236, Meteoritics and Planetary Science, in press, doi: 10.1111/maps.13560, 2020.

Martins, Z., Modica, P., Zanda, B., and L. L. D'Hendecourt, The amino acid and hydrocarbon contents of the Paris meteorite: Insights into the most primitive CM chondrite. Meteoritics and Planetary Science 50, 926-943, 2015. 